\title{
RELATIVISTIC SPINOR DYNAMICS INDUCING THE EXTENDED LORENTZ-FORCE-LIKE EQUATION.
}

\author{
Andreas Bette \\ The Royal Institute of Technology, KTH Syd, Campus Telge, \\ Mariekällgatan 3, S-151 81 Södertälje, Sweden. \\ e-mail: bette@kth.se \\ Jesus Buitrago \\ Department of Astrophysics of the University of La Laguna, \\ C/ Via Lactea, s/n, 38205, La Laguna, Tenerife, Spain. \\ e-mail: jbg@ll.iac.es
}

\begin{abstract}
The special relativistic dynamical equation of the Lorentz force type can be regarded as a consequence of a succession of space-time dependent infinitesimal Lorentz transformations as shown by one of us 7 and discussed in the introduction below. Such an insight indicates that the Lorentz-force-like equation has an extremely fundamental meaning in physics. In this paper we therefore present a set of dynamical Weyl spinor equations inducing the extended Lorentzforce-like equation in the Minowski space-time. The term extended refers to the dynamics of some additional degrees of freedom that may be associated with the classical spin namely with the dynamics of three space-like mutually orthogonal four-vectors, all of them orthogonal to the linear four-momentum of the object under consideration.
\end{abstract}

\section{INTRODUCTION.}

Some years ago it has been noticed by J. Buitrago in [7] that Lorentz force equation may be regarded as a consequence of the action of infinitesimal Lorentz transformations on the velocity four-vector of a relativistic particle, where the parameters of the infinitesimal Lorentz transformations (i.e. of infinitesimal boosts and infinitesimal rotations) are regarded as functions of the position of the particle and not just constants. If these infinitesimal parameters are identified with the components of an external electromagnetic field (evaluated at the four-position $x$ of the particle) multiplied by the infinitesimal lapse of the particle's proper time then the Lorentz force equation will follow automatically.

Let us reassume Buitrago's contribution. Consider the infinitesimal Lorentz transformation in the Minkowski four-vector space $M_{v}$ :

$$
u^{a}(s+\Delta s)=\left[\delta_{b}{ }^{a}+\Delta L_{b}^{a}(x(s))\right] u^{b}(s),
$$


where $u^{a}$ is a time-like or space-like Lorentz four-vector and where $x(s)$, in the Minkowski space-time $M$, denotes a trajectory labelled by a parameter $s$.

The infinitesimal Lorentz transformation in (1.1) is defined by:

$$
\Delta L_{b}^{a}(x(s)):=\left[\frac{1}{2} \alpha^{c d}\left(x^{k}(s)\right) M_{c d}^{a}{ }_{b}\right] \Delta s
$$

where $a, b, c, d$ indices refer to the Lorentz four-vector and four-tensor character of the introduced quatities, so that e.g. $\delta_{b}{ }^{a}$ is the Lorentz identity operator (matrix) etc.. The six generators of the Lorentz transformations $M_{c d}{ }^{a}{ }_{b}$ are, in the Minkowski four-vector space, defined by the following constant Lorentz tensor of fourth $\operatorname{rank}^{1}$ :

$$
M_{c d}^{a}{ }_{b}=-M_{d c}^{a}{ }_{b}:=\delta_{c}^{a} \eta_{d b}-\delta_{d}^{a} \eta_{c b}
$$

where $\eta_{k l}$ denotes the Minkowski metric. $\alpha^{c d}\left(x^{a}(s)\right) \Delta s=-\alpha^{d c}\left(x^{a}(s)\right) \Delta s$ represent the infinitesimal parameters of the infinitesimal Lorentz transformations changing continuously along the trajectory $x(s)$, while $\Delta s$ measures the lapse of the parameter $s$ along the trajectory. Using (1.3) it is easy to see that:

$$
\frac{1}{2} \alpha^{c d}\left(x^{k}(s)\right) M_{c d}{ }^{a}{ }_{b}=\frac{1}{2} \alpha^{c d}\left(x^{k}(s)\right)\left(\delta_{c}{ }^{a} \eta_{d b}-\delta_{d}{ }^{a} \eta_{c b}\right)=\alpha_{b}^{a}\left(x^{k}(s)\right) .
$$

Therefore the equation in (1.1) may be now written as:

$$
\frac{u^{a}(s+\Delta s)-u^{a}(s)}{\Delta s}=\alpha^{a}{ }_{b}\left(x^{k}(s)\right) u^{b}
$$

where the identity in (1.4) has been used. Taking the limit $\Delta s \rightarrow 0$ in (1.5) gives:

$$
\frac{d u^{a}}{d s}=\alpha^{a}{ }_{b}\left(x^{k}(s)\right) u^{b}
$$

It is easy to see from (1.6) that the necessarily non-zero Lorentz norm of the four-vector $u^{a}$ is preserved ${ }^{2}$ along the trajectory $x(s)$.

Two additional assumptions about $u^{a}$ namely that it is a time-like four-vector and that it represents a four-velocity of a physical massive system with a well-defined four-position $x$ in $M$ amounts to ${ }^{3}$ the following conditions:

$$
u^{a}=\frac{d x^{a}}{d s}, \quad u^{a} u_{a}=1
$$

The two equations in (1.6)-(1.7) can be regarded as a second order Lorentz-force-like differential equation:

$$
\frac{d^{2} x^{a}}{d s^{2}}=\alpha^{a}{ }_{b}\left(x^{k}\right) \frac{d x^{b}}{d s} .
$$

\footnotetext{
${ }^{1}$ as well-known from quantum mechanics the same generators multiplied by a purely imaginary number e.g. $i:=\sqrt{-1}$ are identified with the intrinsic spin one angular four-momentum operator.

${ }^{2}$ because of $\alpha^{\prime}$ s antisymmetric Lorentz tensor character.

${ }^{3}$ in this paper the signature convention of the Lorentz metric in the Minkowski space $M$ and the corresponding Minkowski vector space $M_{v}$ is +--- .
} 
whose solutions are the assumed trajectories $x(s)$ in $M$, while the parameter $s$ is then recognised as the proper time of the system with the four-velocity $u^{a}$.

If we, in addition, assume that the function $\alpha$, defining the infinitesimal parameters of the infinitesimal Lorentz transformations as given in (1.1) and (1.2), is proportional to the external electromagnetic field present along the system's trajectory in the following simplest possible way:

$$
\alpha^{c d}\left(x^{m}(s)\right):=\frac{e}{m} F^{c d}\left(x^{m}(s)\right)
$$

then the equation in (1.8) is not only Lorentz-force-like but becomes exactly the Lorentz force equation.

The classical dynamical principle leading to the equation in (1.6) where $u^{a}$ is a time-like or a space-like Lorentz four-vector following a trajectory in $M$ is very simple and fully geometrical. The key point for its validity is the identity proved in (1.4).

At the classical relativistic level that we discuss in this paper there is in this dynamical principle nothing to tell us how to choose a second rank antisymmetric Lorentz tensor valued function $\alpha$ in (1.2). We made a choice in (1.9) and that together with the two assumptions about $u^{a}$ in (1.7) produced the Lorentz force equation. However, whatever choice and assumptions we make, quite generally, we get (1.6) from purely geometrical considerations.

In order to be able to follow the ideas presented in the sequel, the reader must have some basic knowledge about Weyl spinors and their relation to the Lorentz tensors at least to the extent as presented on the first pages of e.g. [1, 10, 11, 12, 13, 15. Basic knowledge of the philosophy behind Penrose's Twistor Theory can also be of value when trying to understand the ideas that led us to the results obtained in this paper.

\section{EXTENDED LORENTZ-FORCE-LIKE EQUATION.}

Consider the four Lorentz invariant, geometrically induced equations such as in (1.6):

$$
\begin{aligned}
\dot{P}^{a} & :=\frac{d P^{a}}{d s}=\alpha^{a b}\left(x^{c}(s)\right) P_{b}, \\
\dot{S}^{a} & :=\frac{d S^{a}}{d s}=\alpha^{a b}\left(x^{c}(s)\right) S_{b}, \\
\dot{V}^{a} & :=\frac{d V^{a}}{d s}=\alpha^{a b}\left(x^{c}(s)\right) V_{b}, \\
\dot{W}^{a} & :=\frac{d W^{a}}{d s}=\alpha^{a b}\left(x^{c}(s)\right) W_{b},
\end{aligned}
$$

where $P$ is time-like while $S, V$ and $W$ are three space-like Lorentz four-vectors following a trajectory $x(s)$ in $M$ and fulfilling the following conditions along the trajectory:

$$
m^{2}:=P^{a} P_{a}=-S^{a} S_{a}=-V^{a} V_{a}=-W^{a} W_{a} \neq 0,
$$


Note that $\mathrm{m}^{2}$, the square of the norm of the time-like four-vector $P$, is a constant of motion. In (2.1)-(2.6) we thus defined dynamics of an orthogonal tetrad of Lorentz four-vectors $P, S, V$ and $W$, following an, as yet unspecified, trajectory in $M$, along which it is infinitesimally Lorentz transformed by an external $\alpha$ field.

Now we make an additional assumption about the time-like four-vector $P$ identifying it with the linear four-momentum of an object following the trajectory $x(s)$ in $M$. Therefore we require additionally:

$$
\dot{x}^{a}:=\frac{d x^{a}}{d s}=\frac{P^{a}}{\sqrt{P^{b} P_{b}}}=\frac{P^{a}}{m},
$$

so that the parameter $s$ may again be recognised as the proper time of the object moving along the trajectory $x(s)$. This fact follows trivially from (2.7) because it implies that $\dot{x}^{a} \dot{x}_{a}=1$.

The equations in (2.1) and in (2.7) define together the (usual) Lorentz-force like equation while the additional equations in (2.2)-(2.4) obeying the conditions displayed in (2.5)(2.6) form the extension of the Lorentz-force-like equation alluded to in the title of this section. This extension defines new degrees of freedom of the object following the trajectory $x(s)$ in $M$. These degrees of freedom may be associated with intrinsic spin of the object. See a short discussion concerning this issue below.

We wish also to stress the fact that the dynamical equations in (2.1)-(2.4), describing dynamics of the four four-vectors $P, S, V$ and $W$, are a consequence of the geometrical considerations as briefly discussed in the introduction [7].

To represent the four-vectors $P, S, V$ and $W$ we will use a pair of non-proportional spinors $\pi$ and $\eta$. By doing so we will automatically fulfil the conditions in (2.5)-(2.6). Let therefore the time-like four-vector $P$ and the three space-like four-vectors $S, V, W$ be defined spinorially [13] in the standard way (abstract index notation in the sense of Penrose [10] is used when appropriate):

$$
\begin{gathered}
P_{a}:=P_{A A^{\prime}}=\pi_{A^{\prime}}(s) \bar{\pi}_{A}(s)+\bar{\eta}_{A}(s) \eta_{A^{\prime}}(s), \\
S_{a}:=S_{A A^{\prime}}=\pi_{A^{\prime}}(s) \bar{\pi}_{A}(s)-\bar{\eta}_{A}(s) \eta_{A^{\prime}}(s), \\
V_{a}:=V_{A A^{\prime}}=\pi_{A^{\prime}}(s) \bar{\eta}_{A}(s)+\bar{\pi}_{A}(s) \eta_{A^{\prime}}(s), \\
W_{a}:=W_{A A^{\prime}}=i\left(\pi_{A^{\prime}}(s) \bar{\eta}_{A}(s)-\bar{\pi}_{A}(s) \eta_{A^{\prime}}(s)\right) .
\end{gathered}
$$

Using the definitions in (2.8)-(2.11), simple spinor algebra calculations show that the conditions in (2.5)-(2.6) are automatically fullfilled. Certain attempts, to formulate the traditional Lorentz force equation by the use of spinor representation of the linear four-momentum $P$ as in (2.8), have been put forward previously [14].

Now we claim that besides this automatic fulfillment of (2.5) $-(2.6)$, all the equations in (2.1)-(2.4) are induced by the following relatively simple dynamical spinor equations:

$$
\frac{d \pi^{A^{\prime}}}{l} \equiv \dot{\pi}^{A^{\prime}}=-c \pi^{A^{\prime}}-b \eta^{A^{\prime}}, \quad \frac{d \eta^{A^{\prime}}}{l} \equiv \dot{\eta}^{A^{\prime}}=a \pi^{A^{\prime}}+c \eta^{A^{\prime}}
$$


where the complex valued Lorentz scalar functions $a, b$ and $c$ are given by:

$$
a=\frac{\alpha^{S^{\prime} T^{\prime}}(x) \eta_{S^{\prime}} \eta_{T^{\prime}}}{\left(\pi^{K^{\prime}} \eta_{K^{\prime}}\right)}, \quad b=\frac{\alpha^{S^{\prime} T^{\prime}}(x) \pi_{S^{\prime}} \pi_{T^{\prime}}}{\left(\pi^{K^{\prime}} \eta_{K^{\prime}}\right)}, \quad c=-\frac{\alpha^{S^{\prime} T^{\prime}}(x) \pi_{S^{\prime}} \eta_{T^{\prime}}}{\left(\pi^{K^{\prime}} \eta_{K^{\prime}}\right)} .
$$

and where the symmetric second rank spinor $\alpha^{S^{\prime} T^{\prime}}(x)$, in the standard manner, represents the given external field $\alpha$ and where $x$ represent events on the object's trajectory in $M$.

If this claim is true then this implies that the equation in (2.12) extends the geometrical principle as described by J. Buitrago in [7] to the space of the two spinors $\pi, \eta$. We now sketch the main parts of the proof showing that (2.12) induces the equations in (2.1)-(2.4):

Multiplying the first equation in (2.12) by $\bar{\pi}^{A}$ and the second by $\bar{\eta}^{A}$ gives:

$$
\begin{gathered}
\bar{\pi}^{A} \dot{\pi}^{A^{\prime}}=\bar{\pi}^{A}\left(-c \pi^{A^{\prime}}-b \eta^{A^{\prime}}\right) \\
\bar{\eta}^{A} \dot{\eta}^{A^{\prime}}=\bar{\eta}^{A}\left(a \pi^{A^{\prime}}+c \eta^{A^{\prime}}\right) .
\end{gathered}
$$

Taking the complex conjugates of (2.14) and (2.15) gives:

$$
\begin{gathered}
\pi^{A^{\prime}} \dot{\bar{\pi}}^{A}=\pi^{A^{\prime}}\left(-\bar{c} \bar{\pi}^{A}-\bar{b} \bar{\eta}^{A}\right), \\
\eta^{A^{\prime}} \dot{\bar{\eta}}^{A}=\eta^{A^{\prime}}\left(\bar{a} \bar{\pi}^{A}+c \bar{\eta}^{A}\right) .
\end{gathered}
$$

Adding the four equation in (2.14), (2.15), (2.16), (2.17) sidewise to each other gives:

$$
\begin{gathered}
\bar{\pi}^{A} \dot{\pi}^{A^{\prime}}+\bar{\eta}^{A} \dot{\eta}^{A^{\prime}}+\text { c.c. }= \\
=-\left[b \bar{\pi}^{A} \eta^{A^{\prime}}+\bar{b} \bar{\eta}^{A} \pi^{A^{\prime}}+(c+\bar{c}) \bar{\pi}^{A} \pi^{A^{\prime}}\right]+\left[a \bar{\eta}^{A} \pi^{A^{\prime}}+\bar{a} \bar{\pi}^{A} \eta^{A^{\prime}}+(c+\bar{c}) \bar{\eta}^{A} \eta^{A^{\prime}}\right] .
\end{gathered}
$$

On the other hand we note that using spinor representation of the equation in (2.1) gives:

$$
\dot{\bar{\pi}}^{A} \pi^{A^{\prime}}+\bar{\pi}^{A} \dot{\pi}^{A^{\prime}}+\dot{\bar{\eta}}^{A} \eta^{A^{\prime}}+\bar{\eta}^{A} \dot{\eta}^{A^{\prime}}=\alpha^{A A^{\prime} B B^{\prime}}(x)\left(\bar{\pi}_{B} \pi_{B^{\prime}}+\bar{\eta}_{B} \eta_{B^{\prime}}\right)
$$

where $\alpha^{A A^{\prime} B B^{\prime}}(x)$ is the spinor equivalent of the antisymmetric Lorentz-tensor $\alpha^{a b}$ in (2.1). Spinor manipulating (2.19) further we note that the external four-force field $\alpha$ in (2.19) may, in the standard way, be represented by ${ }^{4}$ :

$$
\alpha^{a b}(x)=-\alpha^{b a}(x)=\alpha^{A A^{\prime} B B^{\prime}}(x)=-\alpha^{B B^{\prime} A A^{\prime}}(x):=\alpha^{A^{\prime} B^{\prime}}(x) \epsilon^{A B}+\text { c.c. } .
$$

\footnotetext{
${ }^{4}$ algebraically in (2.20) the force field is represented by either an antisymmetric Lorentz tensor of second rank or an hermitian spinor of fourth rank twice primed and twice unprimed or equivalently by a symmetric spinor of second rank either unprimed or primed, all these representations being physically equivalent. We use therefore the same generic letter $\alpha$ for these quantities.
} 
Now we decompose the spinor $\alpha^{A^{\prime} B^{\prime}}$ as follows:

$$
\alpha^{A^{\prime} B^{\prime}}(x)=\frac{a}{\pi^{C^{\prime}} \eta_{C^{\prime}}} \pi^{A^{\prime}} \pi^{B^{\prime}}+\frac{c}{\pi^{C^{\prime}} \eta_{C^{\prime}}} \pi^{A^{\prime}} \eta^{B^{\prime}}+\frac{c}{\pi^{C^{\prime}} \eta_{C^{\prime}}} \eta^{A^{\prime}} \pi^{B^{\prime}}+\frac{b}{\pi^{C^{\prime}} \eta_{C^{\prime}}} \eta^{A^{\prime}} \eta^{B^{\prime}}
$$

with $a, c$, and $b$ being defined as in (2.13). Using the decomposition in (2.21) we may rewrite (2.19) according to ${ }^{5}$ :

$$
\begin{gathered}
\bar{\pi}^{A} \dot{\pi}^{A^{\prime}}+\bar{\eta}^{A} \dot{\eta}^{A^{\prime}}+\text { c.c. }= \\
=-\left[b \bar{\pi}^{A} \eta^{A^{\prime}}+\bar{b} \bar{\eta}^{A} \pi^{A^{\prime}}+(c+\bar{c}) \bar{\pi}^{A} \pi^{A^{\prime}}\right]+\left[a \bar{\eta}^{A} \pi^{A^{\prime}}+\bar{a} \bar{\pi}^{A} \eta^{A^{\prime}}+(c+\bar{c}) \bar{\eta}^{A} \eta^{A^{\prime}}\right],
\end{gathered}
$$

proving our assertion that (2.1) is induced by (2.12). This follows simply from the fact that (2.22) and (2.18) are identical.

By imitating the above steps, using the spinor representations of $S, V$, and $W$, as defined in (2.9), (2.10), (2.11) it may be shown that also the equations in (2.2), (2.3), (2.4) are all induced by the same dynamical spinor equations in (2.12). We call therefore these equations the "master equations". This completes the proof that (2.1), (2.2), (2.3), (2.4) are induced by (2.12) while the conditions in (2.5)-(2.6) are automatically fulfilled due to the definitions in (2.8)-(2.11).

If we require in addition that (2.7), which when written spinorially, reads :

$$
\dot{x}^{A A^{\prime}}:=\frac{d x^{A A^{\prime}}}{d s}=\frac{\pi^{A^{\prime}} \bar{\pi}^{A}+\bar{\eta}^{A} \eta^{A^{\prime}}}{\sqrt{2\left(\bar{\pi}^{B} \bar{\eta}_{B}\right)\left(\pi^{B^{\prime}} \eta_{B^{\prime}}\right)}}=\left(\frac{P^{A A^{\prime}}}{m}\right),
$$

is valid with $x$, as always, denoting points (events) along the object's trajectory in space-time, then the parameter $s$ is once again recognised as the object's proper time parameter while $P$ denotes its linear momentum four-vector.

The $S, V$ and $W$ in (2.9)-(2.11) may be thought of as defining the axis of inertia rigidly attached to an object with the linear four-momentum $P$. If so, then any space-like four-vector, formed as a linear combination of $S, V$ and $W$ (divided by $m$ by obvious dimensional requirements) defines the, so called, Pauli-Lubański spin four-vector. Now, quick glance at any equation arising as any such linear combination of the equations in (2.9)-(2.11) and at the equation (11.164) in 8 reveals that the two equations are proportional to each other only if the gyromagnetic ratio $g$ is equal to two. The "master equations" in (2.12) together with the requirement in (2.23) may thus be regarded as a classical relativistic limit of the equations of motion that describe dynamics of the (classical limit of the) spinning electron $(g=2)$ with any constant real value of its spin i.e. with any value of the norm of its Pauli-Lubański spin four-vector.

Note that the function defined by the positive real valued Lorentz scalar function:

$$
P^{a} P_{a}=2\left(\bar{\pi}^{A} \bar{\eta}_{A}\right)\left(\pi^{A^{\prime}} \eta_{A^{\prime}}\right)=m^{2},
$$

\footnotetext{
${ }^{5}$ c.c. is short hand notation for complex conjugation.
} 
should then be identified with the square of the rest mass of the object (particle) while the function:

$$
m=\sqrt{2}\left|\bar{\pi}^{A} \bar{\eta}_{A}\right|=\sqrt{2}\left|\pi^{A^{\prime}} \eta_{A^{\prime}}\right|
$$

defines its positive rest mass.

Note also that "master equations" in (2.12) imply that not only the function ${ }^{6} m \neq 0$, in (2.25), is a constant of motion but that entire complex valued Lorentz scalar function:

$$
f:=\pi^{A^{\prime}} \eta_{A^{\prime}}=f_{0} \neq 0
$$

is also a constant of motion. This may be easily proved because by contracting the first equation in (2.12) with the spinor $\eta$ and the second with the spinor $\pi$ :

$$
\begin{gathered}
\eta_{A^{\prime}} \dot{\pi}^{A^{\prime}}=-c f, \\
\pi^{A^{\prime}} \dot{\eta}_{A^{\prime}}=c f,
\end{gathered}
$$

and by adding (2.27) and (2.28) to each other, we find that the nonvanishing complex valued function $f$ in (2.26) fulfils:

$$
\dot{f}=0 \text { i.e. } f=f_{0}=\text { const. }\left|f_{0}\right|>0 \text {. }
$$

In the next section we show explicitly how the "master equations" (2.12) can be integrated in the case when the "magnetic" and "electric" fields are constant, equally valued and perpendicular to each other and in the case of constant "electric" field or constant "magnetic" field or both of them constant and being parallel to each other (in some laboratory frame).

The obtained trajectories will, off course, be the very well known ones, see e.g. 9]. However we get some additional information about the motion of the remaining legs of the tetrad attached to our dynamical object. This may be interpreted as precession of the intrinsic spin vector (including the kinematic Thomas precession [8]) attached to the object ${ }^{7}$.

Coping with the non-constant external $\alpha$ field is much more difficult and we present spinor equations for this general case in the last section of this paper.

\section{TWO EXAMPLES AND THEIR SOLUTIONS.}

Concrete constructions of solutions require a choice of the external field and a choice of a suitable frame (corresponding to a laboratory frame in the Minkowski space-time) in the spinor space $S$. The basis in such a fixed (inertial) spin-frame (see e.g. [13, 15]) will be denoted by $(\iota, o)$ :

\footnotetext{
${ }^{6}$ and thereby the function $m^{2}$ in (2.24).

7 an alternative classical limit of the dynamics (of a "charged" relativistic spinning and massive object) that starts from a twistor phase space formulation and the second order formulation of the minimally coupled Dirac equation has been introduced in [1] 2].
} 


$$
\iota_{A}, o_{A} \text { and } \bar{\iota}_{A^{\prime}}, \bar{o}_{A^{\prime}}
$$

and normalized by the requirement:

$$
\iota^{A} O_{A}=1, \bar{\iota}^{A^{\prime}} \bar{o}_{A^{\prime}}=1 \text {. }
$$

The two dynamical spinors $\pi, \eta$ may now, with respect to the chosen fixed spin frame, be expressed by means of their components:

$$
\begin{gathered}
\pi^{A^{\prime}}=u \bar{\iota}^{A^{\prime}}-z \bar{o}^{A^{\prime}}, \quad \eta^{A^{\prime}}=v \bar{\iota}^{A^{\prime}}-w \bar{o}^{A^{\prime}}, \\
\bar{\pi}^{A}=\bar{u} \iota^{A}-\bar{z} O^{A}, \quad \bar{\eta}^{A}=\bar{v} \iota^{A}-\bar{w} o^{A},
\end{gathered}
$$

where, in order to diminish the abundance of the indices, for the components of the two spinors $\pi$ and $\eta$ we introduced the notation:

$$
\begin{gathered}
\pi^{A^{\prime}} \bar{o}_{A^{\prime}}=u, \quad \pi^{A^{\prime}} \bar{\iota}_{A^{\prime}}=z, \quad \eta^{A^{\prime}} \bar{o}_{A^{\prime}}=v, \quad \eta^{A^{\prime} \bar{\iota}_{A^{\prime}}=w .} \\
\bar{\pi}^{A} o_{A}=\bar{u}, \quad \bar{\pi}^{A} \iota_{A}=\bar{z}, \quad \bar{\eta}^{A} o_{A}=\bar{v}, \quad \bar{\eta}^{A} \iota_{A}=\bar{w} .
\end{gathered}
$$

Using these coordinates the "master equations" in (2.12) become:

$$
\dot{z}=-c z-b w, \quad \dot{w}=a z+c w, \quad \dot{u}=-c u-b v, \quad \dot{v}=a u+c v,
$$

while the spinor product defining the function $f$ (see (2.26)-(2.29) $)$ which is a constant of motion reads:

$$
f=z v-u w=f_{0}=\text { constant } \neq 0
$$

The external Lorentz-force-like field $\alpha$ is, with respect to the chosen spin frame $(\iota, o)$ given by (see e.g. [15] p. 91):

$$
\alpha^{A^{\prime} B^{\prime}}(x)=\alpha_{0}(x) \bar{\iota}^{A^{\prime}} \bar{\iota}^{B^{\prime}}-2 \alpha_{1}(x) \bar{\iota}^{\left(A^{\prime}\right.} \bar{o}^{\left.B^{\prime}\right)}+\alpha_{2}(x) \bar{o}^{A^{\prime}} \bar{o}^{B^{\prime}}
$$

where we have:

$$
\begin{gathered}
\alpha_{0}(x)=\frac{\left(E_{x}-B_{y}\right)+i\left(E_{y}+B_{x}\right)}{2} \\
\alpha_{1}(x)=-\frac{E_{z}+i B_{z}}{2}, \quad \alpha_{2}(x)=-\frac{\left(E_{x}+B_{y}\right)-i\left(E_{y}-B_{x}\right)}{2}
\end{gathered}
$$

with $\vec{E}(x)=\left(E_{x}, E_{y}, E_{z}\right)$ and $\vec{B}(x)=\left(B_{x}, B_{y}, B_{z}\right)$ representing the applied Lorentzforce-like field ${ }^{8}$. Therefore from (3.9) it follows that the functions in (2.13) are, in the chosen frame, given by:

${ }^{8}$ Note that, in this context, our "electric" $(\vec{E})$ and "magnetic" $(\vec{B})$ fields have absorbed the factor $\frac{e}{m}$. In that way the dimension of the field $\alpha$, in natural units, becomes inverse of the time. 


$$
\begin{gathered}
a=\frac{\alpha_{0} w^{2}-2 \alpha_{1} v w+\alpha_{2} v^{2}}{f_{0}}, \\
b=\frac{\alpha_{0} z^{2}-2 \alpha_{1} u z+\alpha_{2} u^{2}}{f_{0}}, \\
c=-\frac{\alpha_{0} z w-\alpha_{1} u w-\alpha_{1} v z+\alpha_{2} u v}{f_{0}} .
\end{gathered}
$$

where $f_{0}$ is the constant of motion obtained in (2.29).

Taking into the account the relation in (2.23) the components of the linear momentum four-vector $P$ are, with respect to the chosen spinor frame (that in the standard way defines the constant tetrad of an inertial frame in the Minkowski four-vector space) given by:

$$
\begin{gathered}
P^{A A^{\prime}} \iota_{A} \bar{\iota}_{A^{\prime}}=\frac{m\left(\frac{d t}{d s}+\frac{d z}{d s}\right)}{\sqrt{2}}=\frac{E+p_{z}}{\sqrt{2}}=z \bar{z}+w \bar{w} \\
P^{A A^{\prime}}{ }_{o_{A} \bar{o}_{A^{\prime}}}=\frac{m\left(\frac{d t}{d s}-\frac{d z}{d s}\right)}{\sqrt{2}}=\frac{E-p_{z}}{\sqrt{2}}=u \bar{u}+v \bar{v} \\
P^{A A^{\prime}}{ }_{O_{A} \bar{\iota}_{A^{\prime}}}=\frac{m\left(\frac{d x}{d s}+i \frac{d y}{d s}\right)}{\sqrt{2}}=\frac{p_{x}+i p_{y}}{\sqrt{2}}=z \bar{u}+w \bar{v}
\end{gathered}
$$

where $m=\sqrt{2 f_{0} \bar{f}_{0}}$ and where $s$ is the proper time of the system with the linear fourmomentum $P$.

The three space-like four vectors $S, V$ and $W$ in (2.9)-(2.11) are, with respect to the chosen spinor frame, given by:

$$
\begin{aligned}
& S^{A A^{\prime}} \iota_{A} \bar{\iota}_{A^{\prime}}=\frac{S_{0}+S_{z}}{\sqrt{2}}=z \bar{z}-w \bar{w}, \quad S^{A A^{\prime}} o_{A} \bar{o}_{A^{\prime}}=\frac{S_{0}-S_{z}}{\sqrt{2}}=u \bar{u}-v \bar{v}, \\
& S^{A A^{\prime}} o_{A} \bar{\iota}_{A^{\prime}}=\frac{S_{x}+i S_{y}}{\sqrt{2}}=z \bar{u}-w \bar{v} \\
& V^{A A^{\prime}} \iota_{A} \bar{\iota}_{A^{\prime}}=\frac{V_{0}+V_{z}}{\sqrt{2}}=z \bar{w}+w \bar{z}, \quad V^{A A^{\prime}} o_{A} \bar{o}_{A^{\prime}}=\frac{V_{0}-V_{z}}{\sqrt{2}}=u \bar{v}+v \bar{u} \\
& V^{A A^{\prime}} o_{A} \bar{\iota}_{A^{\prime}}=\frac{V_{x}+i V_{y}}{\sqrt{2}}=z \bar{v}+w \bar{u} \\
& W^{A A^{\prime}} \iota_{A} \bar{\iota}_{A^{\prime}}=\frac{W_{0}+W_{z}}{\sqrt{2}}=i(z \bar{w}-w \bar{z}), \quad W^{A A^{\prime}} o_{A} \bar{o}_{A^{\prime}}=\frac{W_{0}-W_{z}}{\sqrt{2}}=i(u \bar{v}-v \bar{u}), \\
& W^{A A^{\prime}} o_{A} \bar{\iota}_{A^{\prime}}=\frac{W_{x}+i W_{y}}{\sqrt{2}}=i(z \bar{v}-w \bar{u})
\end{aligned}
$$

If the external field $\alpha$ is constant i.e. does not depend on the position $x$ in $M$ then we may separate two cases: the first case when the "electric" and "magnetic" parts 
the solution of the problem 2 on page 58 in [9]) and the second case when the only non-vanishing part of the $\alpha$ field is the "electric" part or only the "magnetic" part or both "electric" and the "magnetic part are non-vanishing and parallel to each other (the mathematics of these three, just mentioned, options is the same so we call it case number two).

We now proceed to consider the case number one. For that reason we note that (3.7) and (3.11)-(3.13) simplify if we introduce the following constants and variables:

$$
\begin{gathered}
\beta_{0}:=\frac{\alpha_{0}}{f_{0}}, \quad \beta_{1}:=\frac{\alpha_{1}}{\alpha_{0}}, \quad \beta_{2}:=\beta_{0}\left(\frac{\alpha_{2}}{\alpha_{0}}-\left(\frac{\alpha_{1}}{\alpha_{0}}\right)^{2}\right), \\
w_{1}:=w-\beta_{1} v, \quad z_{1}:=z-\beta_{1} u,
\end{gathered}
$$

The expressions in (3.11)-(3.13) and in (3.8) now read:

$$
a=\beta_{0}\left(w_{1}\right)^{2}+\beta_{2} v^{2}, \quad b=\beta_{0}\left(z_{1}\right)^{2}+\beta_{2} u^{2}, \quad c=-\beta_{0} z_{1} w_{1}-\beta_{2} u v, \quad f_{0}=z_{1} v-u w_{1} .
$$

We note further that:

$$
\beta_{2}=0 \text { implies that } \vec{E} \cdot \vec{B}=|\vec{E}|^{2}-|\vec{B}|^{2}=0 \text {. }
$$

By putting $\beta_{2}=0$ the expressions for the first three functions in (3.25) become considerably simplified and we obtain:

$$
a=\beta_{0}\left(w_{1}\right)^{2}, \quad b=\beta_{0} z_{1}^{2}, \quad c=-\beta_{0} z_{1} w_{1} .
$$

The dynamical equations in (3.7) acquire now a form that is very easy to solve:

$$
\begin{gathered}
\frac{d\left(z_{1}+\beta_{1} u\right)}{d s}=-\alpha_{0} \beta_{1} z_{1}, \frac{d\left(w_{1}+\beta_{1} v\right)}{d s}=-\alpha_{0} \beta_{1} w_{1}, \\
\frac{d u}{d s}=-\alpha_{0} z_{1}, \quad \frac{d v}{d s}=-\alpha_{0} w_{1} .
\end{gathered}
$$

Inserting the equations in (3.29) into the equations in (3.28) yields:

$$
\frac{d z_{1}}{d s}=0, \quad \frac{d w_{1}}{d s}=0
$$

Therefore for the constant external $\alpha$ field fulfilling the condition in (3.26) the following simple solutions for the coordinates of the two spinors are obtained fom (3.29)-(3.30):

$$
\begin{gathered}
v=v_{0}-\alpha_{0} w_{10} \cdot s, \quad u=u_{0}-\alpha_{0} z_{10} \cdot s \\
w=w_{10}+\beta_{1} v_{0}-\alpha_{1} w_{10} \cdot s, \quad z=z_{10}+\beta_{1} u_{0}-\alpha_{1} z_{10} \cdot s .
\end{gathered}
$$

where the four complex numbers $z_{10}, w_{10}, u_{0}, v_{0}$ are constants of the spinor motion.

We choose now the $y$ axis along the "electric" field and the $z$ axis along the "magnetic" 


$$
\alpha_{1}=-\frac{i B}{2}, \quad \alpha_{0}=\frac{i B}{2}, \quad \alpha_{2}=\frac{i B}{2}, \quad \beta_{1}=\frac{\alpha_{1}}{\alpha_{0}}=-1,
$$

while the solutions in (3.31)-(3.32) may now be written as follows:

$$
\begin{gathered}
v=v_{0}-\frac{i B}{2} w_{10} \cdot s, \quad u=u_{0}-\frac{i B}{2} z_{10} \cdot s \\
w=w_{10}-v_{0}+\frac{i B}{2} w_{10} \cdot s, \quad z=z_{10}-u_{0}+\frac{i B}{2} z_{10} \cdot s .
\end{gathered}
$$

Forming the components of the linear momentum $P$ in (2.8) in the chosen frame (using (3.14)-(3.16) ) out of the spinor components in (3.34)-(3.35) gives:

$$
\begin{gathered}
\frac{E+p_{z}}{\sqrt{2}}=\left(z_{10}-u_{0}\right)\left(\bar{z}_{10}-\bar{u}_{0}\right)+\left(w_{10}-v_{0}\right)\left(\bar{w}_{10}-\bar{v}_{0}\right)+ \\
+\frac{i B}{2}\left(\bar{z}_{10} u_{0}-z_{10} \bar{u}_{0}+\bar{w}_{10} v_{0}-w_{10} \bar{v}_{0}\right) \cdot s+\frac{B^{2}}{4}\left(\bar{z}_{10} z_{10}+w_{10} \bar{w}_{10}\right) \cdot s^{2} \\
\frac{E-p_{z}}{\sqrt{2}}=\left(v_{0} \bar{v}_{0}+u_{0} \bar{u}_{0}\right)+\frac{i B}{2}\left(\bar{z}_{10} u_{0}-z_{10} \bar{u}_{0} \bar{w}_{10} v_{0}-w_{10} \bar{v}_{0}\right) \cdot s+\frac{B^{2}}{4}\left(\bar{z}_{10} z_{10}+w_{10} \bar{w}_{10}\right) \cdot s^{2}, \\
\frac{p_{x}+i p_{y}}{\sqrt{2}}=\left(\bar{u}_{0} z_{10}-u_{0} \bar{u}_{0}+\bar{v}_{0} w_{10}-v_{0} \bar{v}_{0}\right)+\frac{i B}{2}\left(\bar{z}_{10} z_{10}+w_{10} \bar{w}_{10}-u_{0} \bar{z}_{10}+\right. \\
\left.+z_{10} \bar{u}_{0}-\bar{w}_{10} v_{0}+w_{10} \bar{v}_{0}\right) \cdot s-\frac{B^{2}}{4}\left(\bar{z}_{10} z_{10}+w_{10} \bar{w}_{10}\right) \cdot s^{2} .
\end{gathered}
$$

Substracting (3.37) from (3.36) gives that $p_{z}$ is a constant of motion:

$$
p_{z}=\frac{\left(z_{10}-u_{0}\right)\left(\bar{z}_{10}-\bar{u}_{0}\right)+\left(w_{10}-v_{0}\right)\left(\bar{w}_{10}-\bar{v}_{0}\right)-\left(v_{0} \bar{v}_{0}+u_{0} \bar{u}_{0}\right)}{\sqrt{2}}=\text { const. }
$$

Adding (3.37) to (3.36) and to (3.38) and to its complex conjugate reveals that $E+p_{x}$ is also a constant of motion:

$$
E+p_{x}=\frac{2 \Re\left(\bar{u}_{0} z_{10}+\bar{v}_{0} w_{10}\right)-\left(\bar{u}_{0} u_{0}+\bar{v}_{0} v_{0}\right)+\left(z_{10}-u_{0}\right)\left(\bar{z}_{10}-\bar{u}_{0}\right)}{\sqrt{2}}=\text { const. }
$$

$\Re$ is short notation for the real part of. The trajectory in space-time is now obtained by a simple integration of (3.36)-(3.38) using the relation between the position four-vector and the linear four-momentum as displayed in (3.14)-(3.16). The trajectories coincide qualitatively with the ones obtained in 9] as they off course should.

Inserting the solutions in (3.34)-(3.35) into the definitions in (2.9)-(2.11) describes the motion (precession) of the tetrad attached to the object. It solves thereby the dynamical equation in (2.2)-(2.4) subject to the condition in (2.5)-(2.6). The precession consists of 
two parts one purely kinematical (the Thomas precession) and one dynamical induced by the "master equation".

We conclude that the first special case has a simple explicit analytical solution.

Now we proceed to analyse the second case when the "magnetic" part and the "electric" part of the $\alpha$ field are parallell to each other or any of them is vanishing. At the end we specilize to the case of constant "magnetic" field that gives a very well-known circular trajectory, however, the "master equations" imply also the precession of the legs of the tetrad in (2.9)-(2.11). Now we proceed to the construction of the explicit solution. Choose therefore the $z$ axis as the common line along which the (possibly) two fields ("magnetic" and "electric") are directed. Then the formulas for the functions in (3.11)(3.12) simplify again and read:

$$
a=-\frac{2 \alpha_{1} v w}{f_{0}}, \quad b=-\frac{2 \alpha_{1} u z}{f_{0}}, \quad c=\frac{\alpha_{1} u w+\alpha_{1} v z}{f_{0}} .
$$

Inserting the functions in (3.39) into the "master equation" in (3.7) and using (3.8) give a trivially simple set of four first order equations for the four components of the two spinors:

$$
\dot{u}=\alpha_{1} u, \quad \dot{z}=-\alpha_{1} z, \quad \dot{v}=\alpha_{1} v, \quad \dot{w}=-\alpha_{1} w .
$$

The solutions of (3.40) are of course given by:

$$
u=u_{0} e^{\alpha_{1} s}, \quad z=z_{0} e^{-\alpha_{1} s}, \quad v=v_{0} e^{\alpha_{1} s}, \quad w=w_{0} e^{-\alpha_{1} s},
$$

where $u_{0}, z_{0}, v_{0}$ and $w_{0}$ are complex valued constants. Inserting (3.41) into the expressions in (3.14)-(3.22) gives final solution for this case. Therefore we may conclude that the second case with the constant external "magnetic" and "electric" field is also explicitly solved.

Exploring the second solution a bit further we note that in the special case of pure constant "magnetic" field along the $z$ axis, since $\alpha_{1}=-i B_{z} / 2$, the integration constants $u_{0}, z_{0}, v_{0}$ and $w_{0}$ may be chosen as real. From (3.18) we find that $S_{x}$ and $S_{y}$ precess around the $z$ axis according to

$$
\begin{aligned}
& S_{x}=\sqrt{2}\left(z_{0} u_{0}-w_{0} v_{0}\right) \cos B_{z} s, \\
& S_{y}=\sqrt{2}\left(z_{0} u_{0}-w_{0} v_{0}\right) \sin B_{z} s,
\end{aligned}
$$

with similar behaviour for $V_{x}, V_{y}, W_{x}$ and $W_{y}$. On the other hand, the $S_{z}, V_{z}$ and $W_{z}$ components remain constant, independent of the strength of the magnetic field, given by

$$
\begin{gathered}
S_{z}=\frac{\sqrt{2}}{2}\left(z_{0}^{2}+v_{0}^{2}-w_{0}^{2}-u_{0}^{2}\right) \\
V_{z}=\sqrt{2}\left(z_{0} v_{0}-u_{0} v_{0}\right), \quad W_{z}=0 .
\end{gathered}
$$




\section{SPINOR DYNAMICS.}

If the external field $\alpha$ is not constant in space-time, the integration of the "master equations" in (2.12) cannot be performed directly, as done in the previous section, because the functions $a, b$ and $c$ depend explicitly on the object's position four-vector $x$. To remedy this, we let the position four-vector of the object be also translated into its spinorial form by the following construction ${ }^{9}$ :

$$
x^{a}:=x^{A A^{\prime}}=h_{1}(s) \pi^{A^{\prime}} \bar{\pi}^{A}+h_{2}(s) \bar{\eta}^{A} \eta^{A^{\prime}}+h_{3}(s) \pi^{A^{\prime}} \bar{\eta}^{A}+\bar{h}_{3}(s) \bar{\pi}^{A} \eta^{A^{\prime}},
$$

where $h_{1}$ and $h_{2}$ are real valued Lorentz, $s$ parameter dependent, scalar functions and where $h_{3}$ is a complex valued Lorentz, $s$ parameter dependent, scalar function.

Contracting $^{10}$ (2.23) with the two spinors $\bar{\eta}$ and $\bar{\pi}$ gives:

$$
\begin{aligned}
\bar{\eta}_{A} \dot{x}^{A A^{\prime}} & =\frac{\bar{f} \pi^{A^{\prime}}}{\sqrt{2\left(\bar{\pi}^{B} \bar{\eta}_{B}\right)\left(\pi^{B^{\prime}} \eta_{B^{\prime}}\right)}}, \\
\bar{\pi}_{A} \dot{x}^{A A^{\prime}} & =-\frac{\bar{f} \eta^{A^{\prime}}}{\sqrt{2\left(\bar{\pi}^{B} \bar{\eta}_{B}\right)\left(\pi^{B^{\prime}} \eta_{B^{\prime}}\right)}} .
\end{aligned}
$$

Taking derivative of (4.1) with respect to $s$ gives:

$$
\begin{aligned}
& \dot{x}^{A A^{\prime}}=\dot{h}_{1}(s) \pi^{A^{\prime}} \bar{\pi}^{A}+\dot{h}_{2}(s) \bar{\eta}^{A} \eta^{A^{\prime}}+\dot{h}_{3}(s) \pi^{A^{\prime}} \bar{\eta}^{A}+\dot{\bar{h}}_{3}(s) \bar{\pi}^{A} \eta^{A^{\prime}}+ \\
& +h_{1}(s) \dot{\pi}^{A^{\prime}} \bar{\pi}^{A}+h_{2}(s) \bar{\eta}^{A} \dot{\eta}^{A^{\prime}}+h_{3}(s) \dot{\pi}^{A^{\prime}} \bar{\eta}^{A}+\bar{h}_{3}(s) \bar{\pi}^{A} \dot{\eta}^{A^{\prime}}+ \\
& +h_{1}(s) \pi^{A^{\prime}} \dot{\bar{\pi}}^{A}+h_{2}(s) \dot{\bar{\eta}}^{A} \eta^{A^{\prime}}+h_{3}(s) \pi^{A^{\prime}} \dot{\bar{\eta}}^{A}+\bar{h}_{3}(s) \dot{\bar{\pi}}^{A} \eta^{A^{\prime}}
\end{aligned}
$$

Inserting the right hand sides of the "master equtions" (2.12) (and their complex conjugates) into (4.4), contracting ${ }^{11}$ the obtained result at first with $\bar{\eta}_{A}$ and thereafter with $\bar{\pi}_{A}$ yield two expressions that can be compared with right hand side of (4.2)-(4.3). By that we obtain the following four first order differential equations for the scalar functions $h_{1}(s), h_{2}(s), h_{3}(s)$ :

$$
\begin{gathered}
\frac{d h_{1}}{d s}-h_{1}(c+\bar{c})+h_{3} \bar{a}+\bar{h}_{3} a=\frac{1}{m}, \quad \frac{d h_{2}}{d s}+h_{2}(c+\bar{c})-\left(h_{3} b+\bar{h}_{3} \bar{b}\right)=\frac{1}{m}, \\
\frac{d h_{3}}{d s}-h_{1} \bar{b}+h_{2} a-h_{3} c+h_{3} \bar{c}=0 .
\end{gathered}
$$

"Master equations" in (2.12) and the equations in (4.5) imply that the parameter $s$ is the proper time of the object. Together they form a closed system of first order

\footnotetext{
${ }^{9}$ this is simply a projection of the position four-vector on the four legs of the dynamical tetrad defined by the four four-vectors $P, S, V$ and $W$.

${ }^{10}$ note that assuming the validity of (2.23) amounts to identification of the parameter $s$ with the proper time of the object.

${ }^{11}$ it is easier first to contract and later to insert the "master equations".
} 
differential equations that induces the equations in (2.1)-(2.4) fulfilling (2.5)-(2.6) and the physical requirement (2.7).

Note that space-time dynamics described in this abstract way (entirely by the equations in (2.12) and (4.5) ) do not make use of the notion of the space-time manifold at all. Position events traced out by a relativistic system become secondary constructions.

\section{SUMMARY AND REMARKS.}

According to the principle presented in [7] and summarised in the introduction, any Lorentz force-like equation can be regarded as a consequence of the geometry of the Minkowski four-vector space. This principle is here extended to the spinor space and allows us to discover a set of coupled spinor equations that describe dynamics of a massive and spinning classical object (with $\mathrm{g}=2$ ). The solutions of these equations describe not only the world trajectories of the object under study but also the degrees of freedom that can be associated to its intrinsic classical (limit of its quantum mechanical discrete) continuous spin values. It would be very interesting to know whether it is possible to find a Lorentz invariant hamiltonian/lagrangian formulation from which the equations in (2.12) and in (4.5) can be derived. If it is possible then a subsequent quantisation procedure would produce a Lorentz invariant first quantised relativistic theory describing a massive, spinning (for any quantised value of the spin) object acted upon by an applied external $\alpha$. These speculations, however, we postpone to future investigations.

\section{ACKNOWLEDGMENTS.}

One of the authors (A.B.) would like to thank "IAC" (Instituto de Astrofisica de Canarias in Tenerife, Spain) for the financial support. He also wishes to thank The Royal Institute of Technology-KTH Syd for providing him with the so called "fakir" funds that freed him from the teaching duties at the time of this research. We also thank Professors Evencio Mediavilla and the members of his group for their hospitality at IAC, where this work was partly carried out and finally accomplished within the frame of the IAC project number $6 / 88$.

\section{References}

[1] Bette Andreas, (2003). Twistors, Special relativity, conformal symmetry and minimal coupling - a review., Reporte de Investigacion No. 26, Septiembre 2003, Universidad de Sonora, Division de Ciencias Exactas y Naturales, Rosales y Blvd. Luis Encinas J., Edif. 3K1, 83000 Hermosillo, Sonora, Mexico.

[2] Bette Andreas, (2000). Twistor Approach to Relativistic Dynamics and to the Dirac Equation. in Clifford Algebras and their Applications in Mathematical Physics, Volume 1: Algebra and Physics Editors: Rafał Abłamowicz, Bernard Fauser, Progress in Physics 18, Birkhäuser, Boston, Basel, Berlin, 75 - 92.

[3] Bette Andreas, (1996). Directly interacting massless particles - a twistor approach, Journal of Mathematical Physics: 7 (4), $1724-1734$.

[4] Bette Andreas, (1993). Twistor phase space dynamics and the Lorentz force equation, Journal of Mathematical Physics: 4 (10), 4617-4627. 
[5] Bette Andreas and Zakrzewski Stanisław, (1997). Extended phase spaces and twistor theory, Journal of Physics A: Mathematical and General: 30 195-209.

[6] Bette Andreas and Zakrzewski Stanisław, (1996). Massive relativistic systems with spin and the two twistor phase space. in The proceedings of the XII-th workshop on "soft" physics, Hadrons-96: Confinement, Novy Svet, Crimea, June 9-16, 1996, National Academy of Sciences of Ukraine, Bogoliubov Institute for Theoretical Physics (Kiev), Simferopol State University (Crimea), Université Claude Bernard de Lyon, Kiev, pp. 336 - 346.

[7] Buitrago Jesús, (1995), Electromagnetic force and geometry of Minkowskian spacetime., Eur. J. Phys. 16, 113-118.

[8] Jackson John David, (1998). Classical Electrodynamics., 3rd Edition ISBN: 0-47130932-X.

[9] Landau Lev and Lifshitz Evgenii Mikhailovich, (1987). Classical theory of fields, Pergamon Press. (reprinted 2000, ISBN 0750627689)

[10] Penrose Roger, (1968). In Batelle rencontres, eds. C.M. de Witt, J.A. Wheeler Princeton University W.A. Benjamin inc. New York, Amsterdam, 135-149.

[11] Penrose Roger, (1967). Twistor Algebra., Journal of Mathematical Physics: 8 (2) 345-366.

[12] Penrose Roger and MacCallum Malcolm A.H., (1972). Twistor theory: an approach to the quantization of fields and space-time., Physics Reports: 6 (4) 241 316.

[13] Penrose Roger and Rindler Wolfgang, (1984) and (1986). Spinors and Space-Time, Cambridge Monographs on Mathematical Physics vol.1 and 2, Cambridge University Press, Cambridge.

[14] Plebański Jerzy (1983) - private communication, Mexico City.

[15] Stewart John, (1990). Advanced General Relativity, Cambridge University Press, Cambridge Monographs on Mathematical Physics. 